\title{
Compliance to Head and Neck Radiotherapy in Our Patient Population
}

\author{
Anup Kumar,MD ${ }^{1}$; Rajanigandha Tudu, $\mathrm{MD}^{2}$; Tannu Kumari,PhD ${ }^{3}$; \\ Susanta Kumar Sahoo, $\mathrm{MSc}^{4}$ \\ ${ }^{1,2,3}$ Rajendra Institute Of Medical Sciences, Ranchi
}

\begin{abstract}
:
Background: The treatment of head and neck cancer is complex, costly, toxic and challenging particularly in this part of the world where majority of the patients are from rural background and lower socioeconomic status. Persistent patient compliance is crucial, unplanned treatment interruptions reduce cure rates. Treatment prolongation reduces local control and overall survival rates, making adherence to treatment a key factor in optimal outcome. The present study estimates the compliance rate in patients who underwent radiotherapy for head and neck cancer.

Materials and Methods: A retrospective study comprising of 67 patients of head and neck cancer registered or referred to the tertiary care centre, RIMS, Ranchi during July '14 to December '16 were taken. The clinical and demographic data were fetched from ODRI: institutional oncology database.

Results: A total of 67 patients were included in the study. The mean age of presentation was 50 years. $73 \%$ of patients were males and $27 \%$ were females. Mostly patients were nontribal $(83.5 \%)$ compared to $16.5 \%$ of tribal patients. $98 \%$ of the patients were married and only $2 \%$ were unmarried. 24 (36\%) of study patients did not miss any treatment; 32 (48\%) patients missed 1 to 6 treatments; 4 (6\%) patients missed 7 to 14 treatments, and $3(4 \%)$ patients missed more than 14 treatments. $3(4 \%)$ patients did not completed the treatment, while 1 (1.5\%) patients did not started the treatment. Reasons for missed treatments were hospitalization and toxicity.

Conclusion: Approximately $84 \%$ of the patients were compliant to cancer-directed therapy. Efforts are ongoing to further improve adherence in our population.
\end{abstract}

\section{Introduction}

Many patients with cancer of head and neck receive treatment with radiotherapy. Radiotherapy has expanded its role in functional organ preservation. Addition of concurrent chemotherapy has improved treatment outcome in these patients. The results of radiation treatment is totally dependent on treatment compliance, which is very challenging in our population. Treatment related toxicities are an important factor impairing treatment compliance, thereby effecting therapeutic outcome. Multiple studies have assessed the clinical impact of unplanned treatment gap. One study estimated a 50\% loss of local control and overall survival when the treatment time exceeded planned duration by more than 14days [3]. Other groups have evaluated loss of local control with increased length of treatment time for various head and neck patients, with a calculated mean reduction in 5 years local control of $1.7 \%$ (range $0.4 \%$ to $2.9 \%$ ) for each day that treatment is prolonged.[5]

In this study, we document compliance rates, common reasons, why treatments are missed and evaluate whether demographic, tumor related and treatment related factors are correlated with treatment compliance in head and neck cancer patients from the institution.

\section{Materials And Methods}

The patients of head and neck malignancy treated with radiotherapy between July 2014 to December 2016 were included in this retrospective study. All records regarding primary malignancy, radiotherapy and chemotherapy were available for review.

\section{Treatment Course:-}

All patients underwent a 5 to 7 weeks course of daily radiation treatment to the primary tumor site and regional lymph nodes. Patients underwent computed tomography simulation in a supine position with face mask immobilization. Radiation was delivered with intensity modulated radiation therapy (IMRT) or threedimensional conformal radiation therapy (3DCRT) . Concurrent chemotherapy was administered in cases that were medically indicated. Radiation dose ranged from 50gy to $70 \mathrm{gy}$ and were prescribed independently of intent to administer chemotherapy. 


\section{Treatment Compliance:-}

Length of radiotherapy treatment course and number of radiotherapy treatment missed were recorded . Reasons for missed treatment were recorded in the patients file by us. For purpose of this study, treatment noncompliance was defined as prolongation of length of treatment by at least 7 calendar days beyond the length of planned treatment course. With a theoretical loss of local control of $1.7 \%$ per unplanned treatment day according to previously published data, this would incur an approximately 10\% reduction in 5 year local control.[4]

The demographic, tumor related and treatment related variables were recorded ; the total number of missed treatment ,age, gender, marital status, education, socio-economic status, baseline performance status according to ECOG scale, nutritional status, primary site of cancer, American joint committee on cancer ( AJCC) tumor stage, radiotherapy technique, concurrent chemotherapy and highest grade acute toxicity. To determine risk factors for non- compliance, the association between any missed treatment (yes or no ) and all categorical variables was assured using the chi square test. Estimated loss of local control was calculated using a projected $1.7 \%$ radiation in control for each unplanned missed treatment day.[4] Public holidays observed at our institution were not counted as missed treatment days.

\section{Measurment Of Acute Toxicity:-}

During the radiotherapy treatment course, patients were evaluated weekly .The severity of acute toxicity symptoms, graded on a scale 1 through 5 , according to common terminology criteria for adverse events version 3.0 were noted. These notes were reviewed and recorded.

\section{Patient \& Tumour Characteristics-}

\section{Results}

Sixty seven HNC patients were identified. The patients were in the age group of 20-76 years . there were 49 males and 18 females in the study group , 33 patients belonged to low socioeconomic group. The most common primary tumor sites were oral cavity $(67 \%)$, Larynx (11\%) and oropharynx (10\%). Majority of the patients had advanced stage disease and the distribution of AJCC stage grouping was I=8 (11.9\%), II=12 $(17.9 \%), \mathrm{III}=21(31.3 \%), \mathrm{IVA}=19(28.4 \%), \mathrm{IVB}=2(3 \%) .10$ patients $(14.9 \%)$ received concurrent chemotherapy .

Table 1: Patient Demographic, Tumor, and Treatment Characteristics

\begin{tabular}{|c|c|c|}
\hline \multirow{2}{*}{\multicolumn{3}{|c|}{ Age at diagnosis }} \\
\hline & & \\
\hline $20-40$ Years & 14 & 20.9 \\
\hline $40-60$ Years & 38 & 56.7 \\
\hline > 60 Years & 15 & 22.4 \\
\hline \multicolumn{3}{|l|}{ Gender } \\
\hline Female & 18 & 26.9 \\
\hline Male & 49 & 73.1 \\
\hline \multicolumn{3}{|l|}{ Marital status } \\
\hline Unmarried & 63 & 94.0 \\
\hline Married & 4 & 6.0 \\
\hline \multicolumn{3}{|l|}{ Race } \\
\hline Tribal & 11 & 16.4 \\
\hline Non Tribal & 56 & 83.6 \\
\hline \multicolumn{3}{|l|}{ Primary tumor site } \\
\hline Oral Cavity & 45 & 67.2 \\
\hline Nasopharynx & 2 & 3.0 \\
\hline Parotid & 3 & 4.5 \\
\hline Oropharynx & 7 & 10.4 \\
\hline Larynx & 8 & 11.9 \\
\hline Paranasal Sinus & 2 & 3.0 \\
\hline \multicolumn{3}{|l|}{ AJCC stage } \\
\hline I & 8 & 11.8 \\
\hline II & 12 & 17.6 \\
\hline III & 21 & 30.9 \\
\hline IV A & 24 & 35.3 \\
\hline IV B & 2 & 2.9 \\
\hline \multicolumn{3}{|l|}{ Radiation Technique } \\
\hline IMRT & 58 & 86.6 \\
\hline 3DCRT & 9 & 13.4 \\
\hline \multicolumn{3}{|l|}{ Concurrent chemotherapy } \\
\hline Yes & 10 & 14.9 \\
\hline No & 57 & 85.1 \\
\hline Weight loss & & \\
\hline
\end{tabular}


Compliance To Head And Neck Radiotherapy In Our Patient Population

\begin{tabular}{llll}
\hline No & & 50 & 74.6 \\
Yes & & 17 & 25.4 \\
& & & \\
Lower & Income & 33 & 48.5 \\
Group & & 29 & 42.6 \\
Middle & Income & 5 & 7.4 \\
Group & & & \\
Higher & Income & \\
Group & & & \\
\hline
\end{tabular}

\section{Treatment Compliance-}

Eight four percent of patient were compliant with the radiotherapy treatment course

(defined as prolonging treatment by fewer than 7 calendar days). 24 patients (36\%) completed radiotherapy with no missed treatment, 32 patients (48\%) missed one to six treatment, 4 patients $(6 \%)$ missed seven to fourteen treatment while 3 patients missed more than 14 treatments (4\%), 3 patients did not complete the treatment (4\%) while 1 patients did not start the ( $1.5 \%)$ treatment.

The most common cause for missed treatment were hospitalization ( $25 \%$ of event ) and toxicity ( $20 \%$ of event ). The reasons for hospitalization were chemotherapy administration, blood transfusion and treatment related toxicity. Other reasons for missed treatment were lack of transportation due to local bandh (10\%), patient refusal $(10 \%)$, and treatment machine breakdown (10\%). $2 \%$ patients missed treatment due to some family emergency situation. $20 \%$ of the patients missed treatment due to unknown reasons.

Toxicity

Grade ( CTCAE Version 3)*

\begin{tabular}{|l|l|l|l|l|}
\hline & I, No.(\%) & II,No(\%) & III,No(\%) & IV,No(\%) \\
\hline Mucositis & $32(48 \%)$ & $21(31 \%)$ & $13(19 \%)$ & \\
\hline Dermatitis & $33(49 \%)$ & $14(21 \%)$ & $7(10 \%)$ & \\
\hline Xerostomia & $38(57 \%)$ & $11(16 \%)$ & & \\
\hline Nausea & $38(57 \%)$ & $7(10 \%)$ & & \\
\hline Hematological Toxicity & $8(12 \%)$ & $12(18 \%)$ & $3(4 \%)$ & \\
\hline
\end{tabular}

*Common Terminology Criteria for Adverse Events version 3.0*

Numerous acute toxicities were observed. The most common grade 3 toxicity were mucositis, dermatitis and hematological toxicity. There were no grade 4 toxicity reported (Table No.2).

\section{Discussion}

Delays in radiotherapy treatment of head and neck carcinomas have resulted in inferior treatment outcome. Protraction of treatment time decreased the rate of loco regional control for head and neck cancer in several retrospective analyses, which is consistent with accelerated tumor regeneration. With conventional daily treatment schedule, using one fraction per day, these studies describe a loss of local control of $0.4 \%$ to $2.9 \%$ for each day the treatment course is prolonged, with an average of $1.7 \%$ day . Loss of local control is primarily attributed to tumour repopulation during a treatment gap.[9,10] Several methods have been proposed to compensate for prolonged treatment time, including treatment on weekend days or treating two fractions in one day to retain both overall treatment time and dose per fraction, adding on missed fraction to the end of the treatment, to retain dose per fraction and total dose while accepting protraction, or increasing dose per fraction while maintaining treatment time.[4] However, each of these methods has drawbacks. Late effects might be enhanced by increasing the fraction size or treating two fractions in one day, if the inter fraction time is short.[2] Our department added the missed fraction to the end of the treatment course at same dose per fraction. Khalil and colleagues investigated treatment compliance in HNC patient participating in four randomized controlled trials through the European organization for Research and treatment of cancer, Princess Margaret hospital and a continuous hyper fractionated accelerated radiotherapy trial.(CHART) $25 \%$ of patient in these trials had delays of 6 days or more.

Although many studies have assessed compliance rate, few have been able to identify specific predictors of non-compliance. Patel and colleagues, evaluated the predictive value of ethnicity, gender, primary tumor site, stage and nodal status, but did not find any significant variable that could predict for noncompliance.[5] Rajni A. Sethi and colleagues, evaluated several demographic, toxicity and treatment variables for reasons for missed treatment, they found patients with percutaneous endoscopic gastrostomy tube were more likely to missed treatment $(\mathrm{P}=.01)$. No other variable showed a significant association with missed treatments.[1] A larger study assessing 5086 head and neck cancer patients enrolled in Medicare, a federal health insurance plan in the United State, reported that $39 \%$ of the patients had prolonged or incomplete treatment. Patients who underwent surgery prior to radiotherapy were more likely to complete uninterrupted 
radiotherapy possibly, due to selection of healthier patients motivated to undergo more rigorous therapy with both surgery and radiation.[15]

We evaluated multiple socio- economic, demographic, tumor related and treatment related variables as risk factor for non-compliance, but no variable was significant enough to predict non-compliance. The relatively small sample size and low number of missed treatments might have precluded finding other factors that predicted compliance. The most frequent reasons for each missed treatment were noted as reported by the patient, treating physician or radiation technologist at the time of patient absence. Missed treatments were most commonly caused by hospitalization, acute toxicity, treatment machine maintenance. As majority of our patients belonged to low socio-economic status, with poor educational background and were noted to be unaware about the importance of the ongoing treatment. Other reasons were patients refusal, local Bandh /strike.

Therefore, there is need to increase awareness about the disease and its treatment to improve treatment outcome. Although prospective studies are required to evaluate risk factor for non-compliance in the population, it is also important for individual institutions to assess site specific weaknesses in health care delivery that impedes treatment compliance.

Table 2: Correlation of Missed Treatment With Demographic, Tumor, and Treatment-Related Variables.

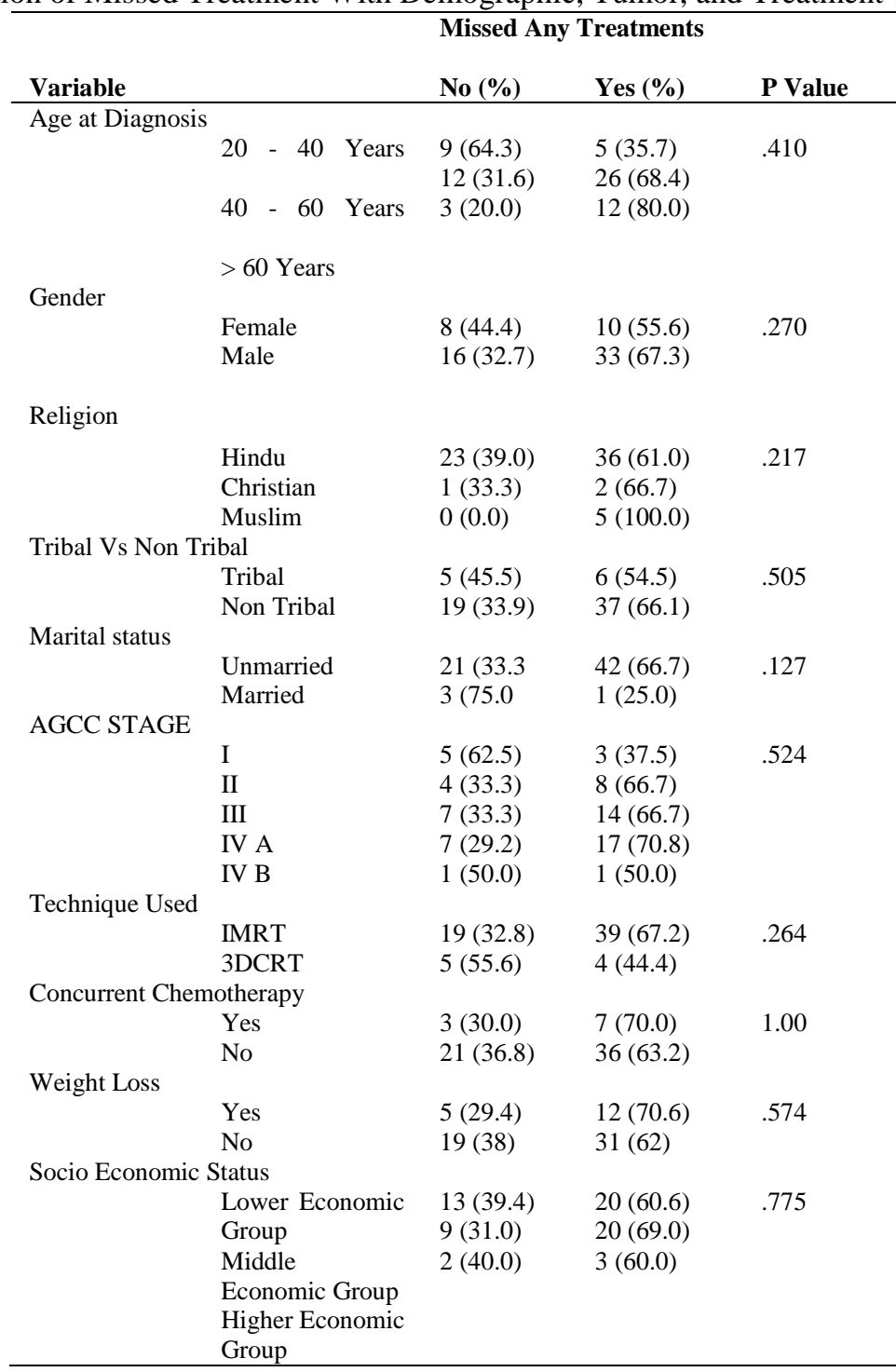

\section{Conclusion}

Definitive radiotherapy for head and neck cancer can be delivered with good adherence in our patient population. Eighty four percent of our treated patients completed treatment with 0 to 6 days of treatment interruption. Further studies are required to evaluate the risk factors for non-compliance. Public awareness about the disease and treatment should be ensured to improve treatment compliance. 


\section{Bibliography}

[1]. Rajni A. sethi, Md; Emily F. Stamell, BS; Leah Price, MS; Mark DeLacure, MD; Nicholas Sanfilippo,MD. Head and neck radiotherapy compliance in an underserved patient population, Laryngoscope 120:july 2010.

[2]. Bese NS, Hendry J, Jeremic B. Effects of prolongation of overall treatment time due to unplanned interruptions during radiotherapy of different tumor sites and practical methods for compensation, Int J radiat Oncol Biol Phys 2007;68:654-661.

[3]. Pajak TF. Laramore GE, Marcial VA, et al. Elapsed treatment days-a critical item for radiotherapy quality control review in head and neck trials: RTOG Report. Int j Radiat oncol Biol Phys 1991;20:13-20.

[4]. Hendry JH, Bentzen SM, Dale RG, et al. A modeled comparison of the effects of using different ways to compensate for missed treatment days in radiotherapy. Clin Oncol 1996;8:297-307.

[5]. Patel UA, Thakakar KH, Holloway N. Patient compliance to radiation for advanced head and neck cancer patients at a teriarry care county hospital. Laryngoscope 2008;118:428-432.

[6]. Maciejewski B,Preuss-Bayer G, Trott KR. The influence of the number of fractions and of overall treatment time on local control and late complication rate in squamous cell carcinoma of the larynx, Int J Radiat oncol Biol Phys 1983;9:321-328.

[7]. Taylor JMG, Withers HR, Mendenhall WM. Dose time considerations of head and neck squamous cell carcinomas treated with irradiation. Radiother oncol 1990;17:95-102.

[8]. Barton MB, Keane TJ, Galla T, et al. The effect of treatment time and treatment interruption on tumour control following radical radiotherapy of laryngeal cancer. Radio-ther Oncol 1992;24:137-143.

[9]. Withers HR, Taylor JM, Maciejewski B. The hazad of accelerated tumor clonogen repopulation during radiotherapy. Acta Oncol 1988;27:131-146.

[10]. Tarnawski R, Fowler J, Skaladowski K, et al. How fast is repopulation of tumor cells during the treatment gap? Int J radiat Oncol Biol Phys 2002;54:229-236.

[11]. Khalil AA, Bentzen SM, Bernier J, et al. Compliance to the prescribed dose and overall treatment time in five randomized clinical trials of altered fractionation in radiotherapy for head and neck carcinomas. Int J Radial Oncol Biol Phys 2003;55:568-575.

[12]. Ban den Bogaert W, Van der Leest A, Rijinders a, Delaere P, Thames H, Van der Schurren E. Dose tumor control decrease by prolonging overall treatment time or interrupting treatment in laryngeal cancer ? Radiother Oncol1995;36:177-182.

[13]. Maciejewski B, Withers HR, Taylor JM, Hiniak A. Dose fractionation and regeneration in radiotherapy for cancer of the oral cavity and oropharynx: tumor dose response and repopulation. Int J Radiat Oncol Biol Phys 1980;16:831-843.

[14]. Bataini JP. Asselain B, Janulerry C, et al, A multivariate primary tumour control analysis in 465 patients treated by radical radiotherapy for cancer of the tonsillar region: Clinical and treatment parameters as prognostic factors. Radiother Oncol 1989;14:265-277.

[15]. Fesinmeyer MD, Mehta V, Tock L, Blough D, McDermott C, ramsey SD, Completionof radiotherapy for local and regional head and neck cancer in Medicare, arch Otolaryngol Head and Neck surg 2009;135:860-867. 